\title{
Dual Metabolomic Platforms Identified a Novel Urinary Metabolite Signature for Hepatitis B Virus-Infected Patients with Depression
}

This article was published in the following Dove Press journal: Diabetes, Metabolic Syndrome and Obesity: Targets and Therapy

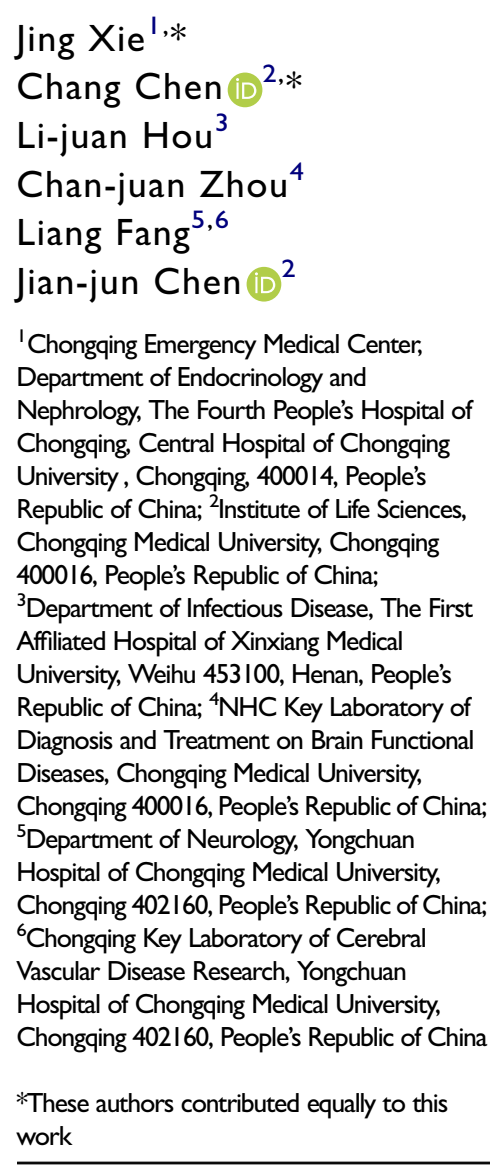

Correspondence: Jian-jun Chen Institute of Life Sciences, Chongqing Medical University, I Yixueyuan Road, Yuzhong District, Chongqing 400016, People's Republic of China

Email chenjianjun@cqmu.edu.cn

Liang Fang

Department of Neurology, Yongchuan Hospital of Chongqing Medical University, 439 Xuanhua Road, Yongchuan District, Chongqing 402I60, People's Republic of China

$\mathrm{Tel} / \mathrm{Fax}+86-23-63664754$

Email fangliang@hospital.cqmu.edu.cn
Objective: Depression could make the treatment outcome worse. However, up to now, no objective methods were developed to diagnose depression in hepatitis B virus (HBV)infected patients. Therefore, the dual metabolomic platforms were used here to identify potential biomarkers for diagnosing HBV-infected patients with depression (dHB).

Methods: Both gas chromatography-mass spectrometry-based and nuclear magnetic resonance-based metabolomic platforms were used to conduct urine metabolic profiling of $\mathrm{dHB}$ subjects and HBV-infected patients without depression (HB). Orthogonal partial leastsquares discriminant analysis was used to identify the differential metabolites between dHB subjects and HB subjects, and the step-wise logistic regression analysis was used to identify potential biomarkers.

Results: In total, 21 important metabolites responsible for distinguishing dHB subjects from HB subjects were identified. Meanwhile, seven potential biomarkers ( $\alpha$-ydroxyisobutyric acid, hippuric acid, azelaic acid, isobutyric acid, malonic acid, levulinic acid, and phenylacetylglycine) were viewed as potential biomarkers. The simplified biomarker panel consisting of these seven metabolites had an excellent diagnostic performance in discriminating dHB subjects from HB subjects. Moreover, this panel could yield a higher accuracy in separating $\mathrm{dHB}$ subjects from $\mathrm{HB}$ subjects than our previous panels (identified by single metabolomic platform) did.

Conclusion: These results suggested that the dual metabolomic platforms could yield a better urinary biomarker panel for $\mathrm{dHB}$ subjects than any single metabolomic platform did, and our results could be helpful for developing an objective method in future to diagnose depression in HBV-infected patients.

Keywords: hepatitis B virus, depression, metabolomics, biomarker

\section{Introduction}

Hepatitis is viewed by Chinese experts as one of the 20 most important and most preventable health problems of China. ${ }^{1}$ Hepatitis B virus (HBV), as the most common cause of hepatitis worldwide, accounts for at least $50 \%$ cases of hepatocellular carcinoma. Currently, although preventable with safe and effective vaccines, there is still about $3 \%$ of the global population chronically infected with HBV. $^{2}$ Currently, no available treatment modalities could completely eliminate HBV from human body. The long-term treatment and economic burden often cause patients to experience negative emotions, such as depressive symptoms. Moreover, HBV-infected patients are prone to suffer from low self-esteem, social 
isolation, hopelessness, and loneliness, thus increasing the risk of developing mental disorders. ${ }^{3}$

Depression is a debilitating mental disorder accompanied by considerable costs, mortality, morbidity, and heightened risk of suicide. It is also viewed as one of the 20 most important and most preventable health problems of China. ${ }^{1}$ This disease is prevalent in chronic HBV-infected patients, partly because it is one of the adverse effects of interferon given against $\mathrm{HBV}^{4}$ Previous studies reported that HBVinfected patients had significantly higher levels of depression compared to healthy controls. ${ }^{5,6}$ Depression could decrease the quality of life of HBV-infected patients. But one of the most serious consequences caused by depression is reducing patients' compliance with prolonged therapeutic regimens. ${ }^{7}$ However, depression is often overlooked in these patients.

In recent years, depression in $\mathrm{HBV}$-infected patients has drawn more and more attention. However, the diagnosis of depression is inadequate as it is only based on subjective identification of symptom clusters rather than objective laboratory tests. This diagnostic modality could cause a considerable error rate. An approach to solve this problem is to identify disease biomarkers for objectively diagnosing depression. Recently, researchers have done many meaningful works to identify potential biomarkers for objectively diagnosing depression. ${ }^{8-11}$ Using metabolomic approaches, we successfully identified some potential urinary biomarkers to diagnose depression in HBV-infected patients. ${ }^{12,13}$ However, the common limitation of these studies is that only one metabolomic platform is used. Currently, three major metabolomic platforms are suited for non-targeted metabolomic mapping: nuclear magnetic resonance (NMR) spectroscopy, gas chromatography (GC)-, and liquid chromatography (LC)-mass spectroscopy (MS). Each one has its advantages and disadvantages. But, none of these metabolomic platforms could provide adequate coverage of the entire human metabonome in a given sample. ${ }^{14}$ Previous studies have reported that the combined use of several metabolomic platforms could substantially enhance the level of metabolome coverage. ${ }^{15,16}$ Our previous study found that the combined application of GC-MS- and NMRbased metabolomic platforms could yield a superior biomarker panel for diagnosing bipolar disorder. ${ }^{17}$ Therefore, in this study, the dual platform approach (NMR spectroscopy and GC-MS) was used to identify a novel urinary metabolite signature for diagnosing depression in HBVinfected patients.

\section{Materials and Methods \\ Patient Recruitment}

Ethical Committee of Chongqing Medical University approved the protocols of this study. HBV-infected patients with depression ( $\mathrm{dHB}$ ) were recruited if they met the following criteria: i) using Hamilton Depression Rating Scale (HDRS) to assess depression severity, and the HDRS score was no less than 17 ; ii) receiving no antidepressant medications; iii) not co-infected with other hepatitis virus or human immunodeficiency virus (HIV); iv) on pre-existing physical or other neuropsychiatric disorders, no alcohol consumption, or illicit drug use; and v) aged 18 and older. The HBV-infected patients without depression (HB) met the following criteria were recruited into this study: i) not co-infected with other hepatitis virus or HIV; ii) on pre-existing physical or neuropsychiatric disorders; iii) no alcohol consumption or illicit drug use; and iv) aged 18 and older. Finally, $81 \mathrm{dHB}$ subjects and $68 \mathrm{HB}$ subjects extracted from our previous studies were included in this study. ${ }^{12,13}$ All patients provided written informed consent, and that this study was conducted in accordance with the Declaration of Helsinki. The detailed information was described in Table 1.

Table I Demographic and Clinical Characteristics of the Included Patients

\begin{tabular}{|l|l|l|l|l|l|l|}
\hline \multirow{2}{*}{ Variables } & \multicolumn{2}{l}{ Training Set } & \multicolumn{2}{l|}{ Testing Set } \\
\cline { 2 - 7 } & HB & dHB & P-value & HB & dHB & P-value \\
\hline Sample size & 46 & 55 & - & 22 & 26 & - \\
Age (years)c & $44.8 \pm 14.5$ & $47.9 \pm 16.6$ & 0.33 & $49.3 \pm 16.4$ & $46.3 \pm 16.7$ & 0.54 \\
Sex (F/M) & $23 / 23$ & $31 / 24$ & 0.50 & $11 / 11$ & $10 / 16$ & 0.43 \\
HDRS & $1.6 \pm 1.1$ & $24.2 \pm 3.8$ & $<0.00001$ & $1.4 \pm 1.2$ & $23.7 \pm 4.1$ & $<0.00001$ \\
BMI & $21.3 \pm 4.0$ & $21.8 \pm 3.7$ & 0.45 & $23.6 \pm 3.8$ & $22.3 \pm 3.2$ & 0.20 \\
\hline
\end{tabular}

Abbreviations: HDRS, Hamilton depression rating scale; $\mathrm{dHB}$, hepatitis B virus-infected patients with depression; $\mathrm{HB}$, hepatitis $B$ virus-infected patients without depression; BMI, body mass index; F, female; $M$, male. 


\section{Metabolites Acquisition}

After overnight fasting, the included patients were told to collect the morning urine samples using a sterile cup. The midstream urine was demanded. The collected urine samples were transferred into a sterile tube and then immediately delivered to the laboratory under low temperature. All urine samples were centrifuged at $1500 \mathrm{~g}$ for $10 \mathrm{~min}$. The obtained supernatant was then divided into equal aliquots. At last, the samples were stored at $-80^{\circ} \mathrm{C}$ until later analysis. In this study, the procedure of urinary metabolites acquisition using GC-MS and NMR spectroscopy was exactly conducted according to our previous studies. ${ }^{10,12,13}$

\section{Data Analysis}

As the contents of metabolites in different urine samples might vary largely, we used the creatinine to normalize the contents of metabolites in different urine samples to control the variation of urine samples. Then, the data would be further scaled to zero mean and unit variance before analyzing. The included patients were randomly assigned to the training set and testing set. Firstly, we used the training set to build orthogonal partial least-squares discriminant analysis (OPLS-DA) model, which was used to show the divergent urinary metabolic phenotypes between $\mathrm{dHB}$ and HB. The 399-iteration permutation test was used to assess whether the built model was over-fit or not. Meanwhile, the testing set was further used to validate the built model.

We used the coefficient loading plot of the built OPLSDA model to find the important metabolites responsible for separating dHB subjects from HB subjects. The correlation coefficient of $|r|>0.258$ (equivalent to a $p$-value of less than 0.05) was chosen as the cut-off value according to the number of samples in the training set. The online software MetaboAnalyst was used to identify the biological functions of these identified important metabolites. Meanwhile, the nonparametric Mann-Whitney $U$-test and Benjamini and Hochberg False Discovery Rate were used to validate the metabolic changes identified by OPLS-DA model. As a small number of urinary metabolites would be more convenient and feasible to diagnose depression, we used the step-wise logistic-regression analysis based on Akaike's information criterion (AIC) rule to optimize the identified important metabolites.

In order to assess the diagnostic performance of the obtained simplified metabolite biomarker panel in diagnosing depression in HBV patients, we performed the receiver- operating characteristic (ROC) curve analysis. The area under the curve (AUC) in both training set and testing set was viewed as an index to quantify the ability of this panel in discriminating dHB subjects from HB subjects. Diagnostic accuracy was categorized as good $(0.8<\mathrm{AUC}<0.9)$ and excellent $(0.9<$ AUC $\leq 1.0) .{ }^{18}$ The SIMCA-P 14.0 (Umetrics, Umeå, Sweden) and SPSS 19.0 were used to conduct data analysis. The Student's $t$-test and Chi-square test were performed to analyze the baseline data. The $p$-value $<0.05$ was considered to be statistically significant.

\section{Results}

\section{Divergent Metabolic Phenotypes}

Firstly, we used the training set to build the OPLS-DA model. The results showed that the $\mathrm{dHB}$ subjects could be obviously separated from HB subjects with no overlap (Figure 1A). The values of two parameters $(\mathrm{R} 2 \mathrm{Y}$ cum $=0.83, \mathrm{Q} 2=0.68)$ were also positive. Meanwhile, the results of 399-iteration permutation test indicated that the built OPLS-DA model was not over-fitted, as all R2- and Q2-values to the left were lower than the original points to the right (Figure 1B). To independently assess the validity of the built OPLS-DA model, the model was used to predict the samples in the testing set. The T-predicted scatter plot showed that all samples were correctly predicted (Figure 2). These results demonstrated that there were divergent metabolic phenotypes between dHB subjects and HB subjects, and the built model held promise as an objective diagnostic method for depression in HBVinfected patients.

\section{Important Urinary Metabolites}

By analyzing the coefficient loading plots of the built model, we found that there were 21 important metabolites responsible for distinguishing $\mathrm{dHB}$ subjects from HB subjects. Compared to $\mathrm{HB}$ subjects, dHB subjects were characterized by higher levels of $\alpha$-hydroxyisobutyric acid, pyruvic acid, acetone, glycine, acetamide, $\beta$-aminoisobutyric acid, glycolic acid, hippuric acid, azelaic acid, dimethylglycine, $\beta$-hydroxybutyric acid, isobutyric acid, D-threitol, levulinic acid, along with lower levels of 2,4-dihydroxypyrimidine, malonic acid, phenylacetylglycine, ethanolamine, methylmalonic acid, hydroxylamine, p-hydroxyphenylacetic acid. The functional analysis found that glyoxylate and dicarboxylate metabolism ( $\mathrm{p}=0.006$, impact $=0.19$ ) and glycine, serine, and threonine metabolism $(\mathrm{p}=0.006$, impact $=0.32)$ were significantly affected (Figure 3). 

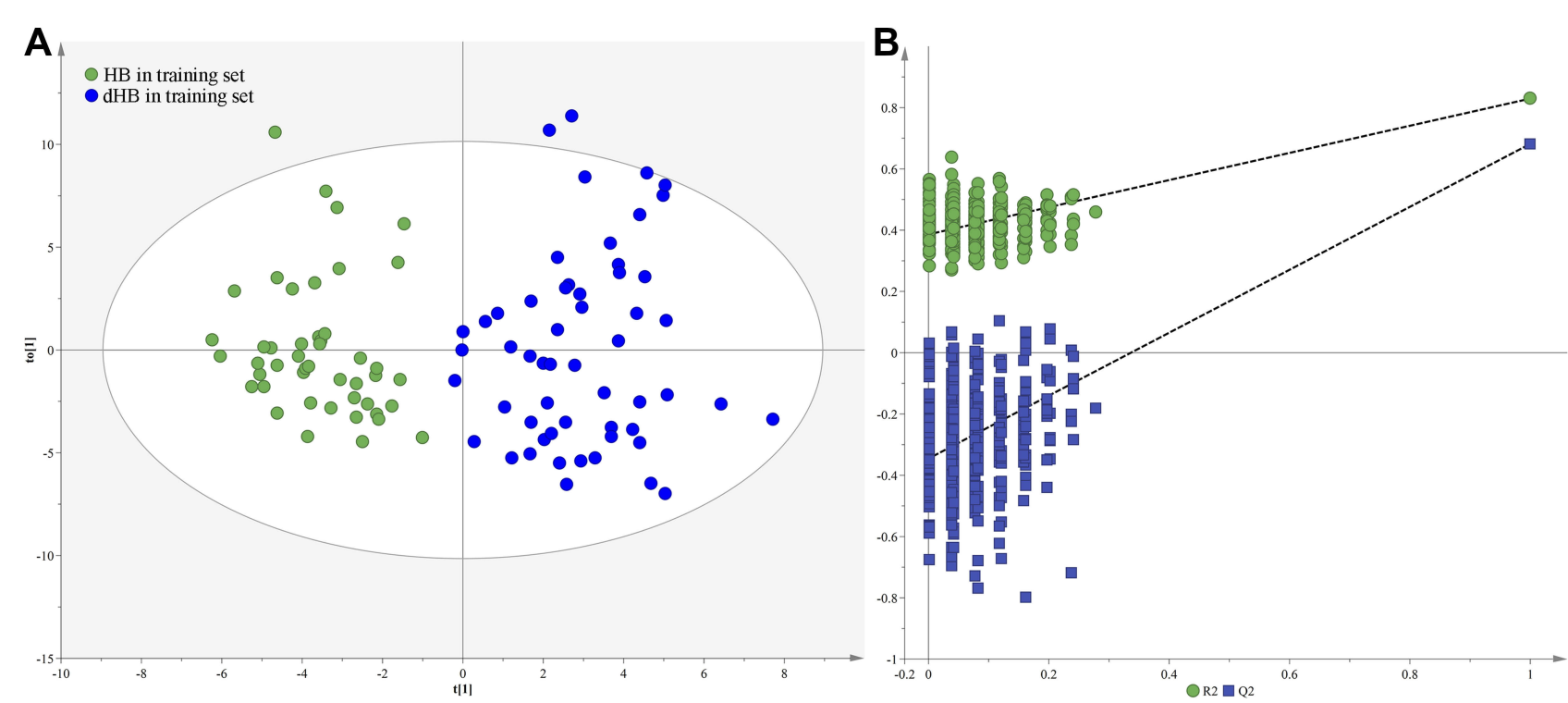

Figure I Metabolomic analysis of urine samples: (A) OPLS-DA model showed a clear discrimination between dHB (blue dot) and HB (green dot) in the training set; (B) permutation test showed all R2- and Q2-values to the left were lower than the original points to the right, demonstrating the OPLS-DA model's robustness.

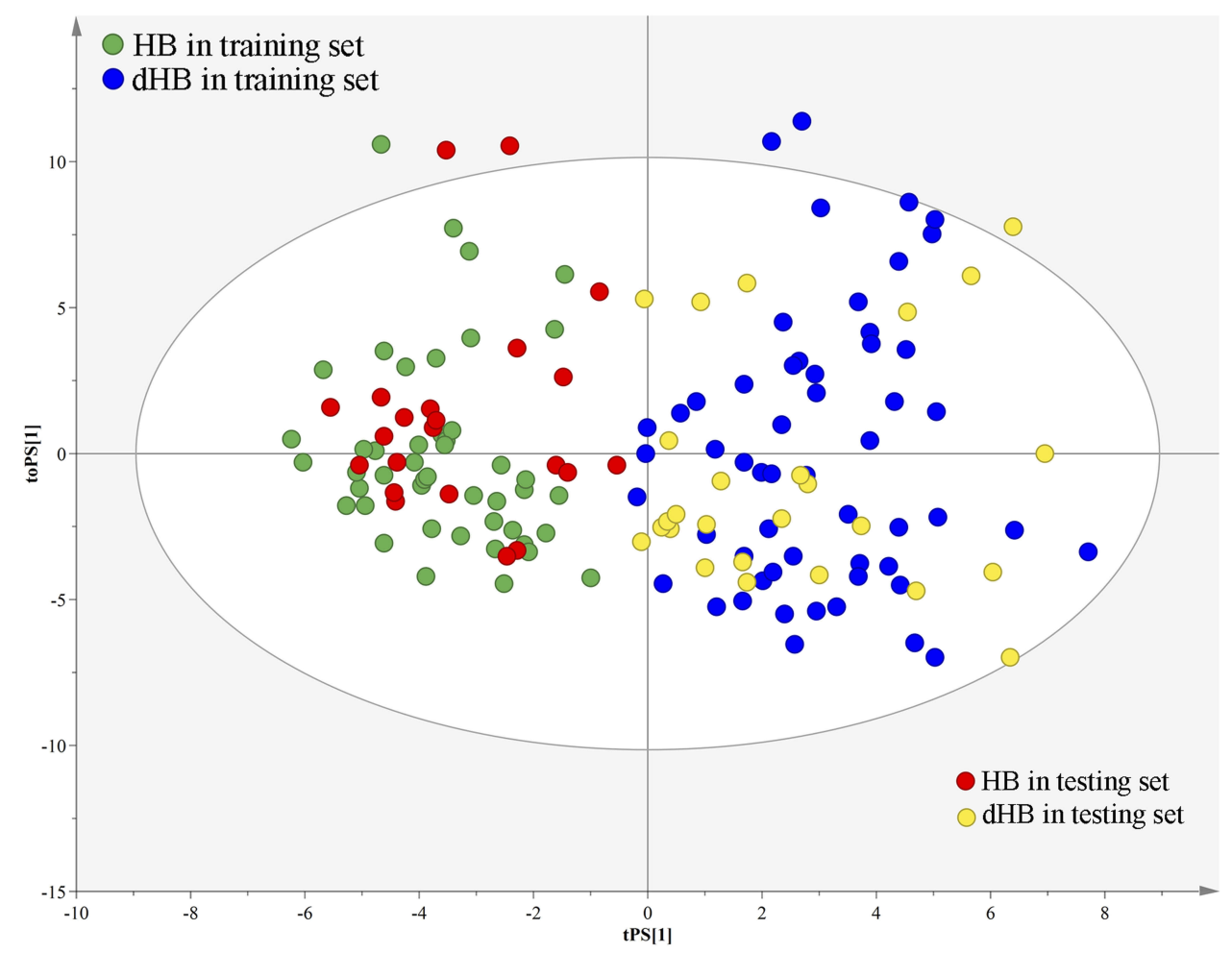

Figure 2 T-predicted scatter plot of OPLS-DA model. The model built with dHB (blue dot) and HB (green dot) in the training set could correctly predict dHB (yellow dot) and $\mathrm{HB}$ (red dot) in the testing set.

\section{Simplified Biomarker Panel}

We used the step-wise logistic-regression analysis based on AIC rule to further analyze the 21 important urinary metabolites. The results showed that the AIC value was minimal when there were seven important urinary metabolites in the logistic-regression model (Figure 4A). The seven important urinary metabolites were $\alpha$-hydroxyisobutyric acid, hippuric acid, azelaic acid, isobutyric acid, malonic acid, levulinic acid, and phenylacetylglycine. Among these identified biomarkers, there were two metabolites (azelaic acid and 


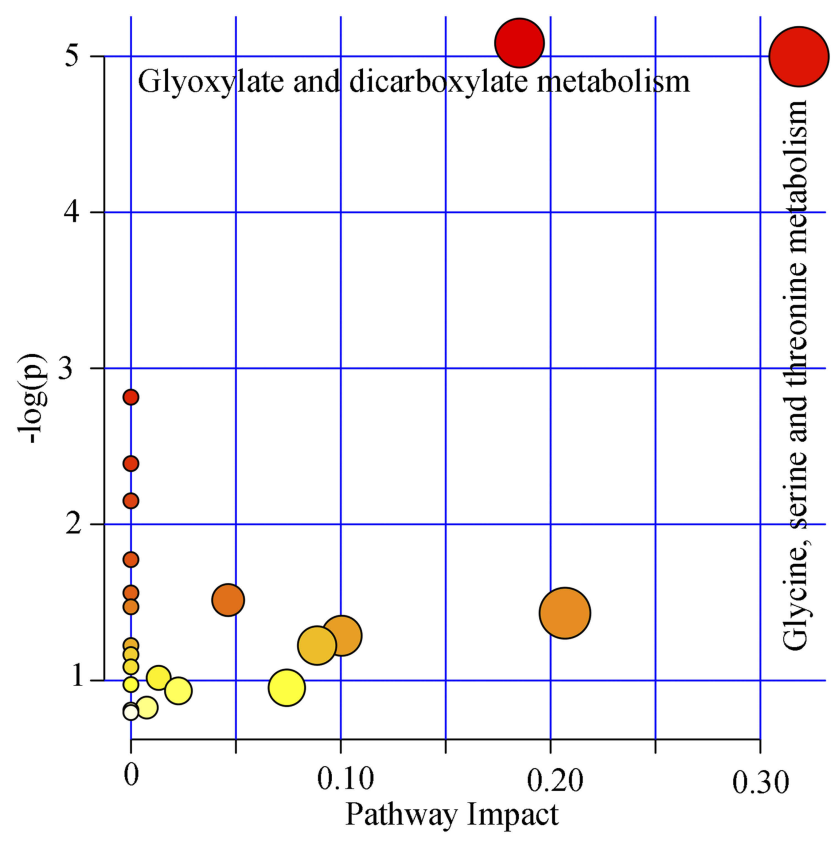

Figure 3 Pathway analysis using the important metabolites. The pathways with $\mathrm{p}<0.05$ and impact value $>0$ were viewed as the significantly affected pathways.

levulinic acid) from GC-MS platform and other five metabolites from NMR platform. The panel consisting of these seven important urinary metabolites could correctly separate dHB subjects and HB subjects with accuracies of $98.0 \%$ in the training set and $95.8 \%$ in the testing set. These results indicated that the most significant deviations between $\mathrm{dHB}$ subjects and HB subjects could be described by these seven important urinary metabolites.

\section{Diagnostic Performance}

The probability of depression in HBV-infected patients could be calculated using the identified simplified biomarker panel:
$\mathrm{P}(\mathrm{Y}=1)=1 /(1+\mathrm{e}-\mathrm{y}) ; \mathrm{y}=-47.837^{*} \alpha$-hydroxyisobutyric acid + $2.416^{*}$ phenylacetylglycine-4.283*hippuric acid-7.665* isobutyric acid $+3.286^{*}$ malonic acid $-1542.158^{*}$ azelaic acid $-484.869 *$ levulinic acid +24.759 . Then, we performed ROC analysis to build a curve using the obtained probabilities. The results showed that the identified simplified biomarker panel could yield an AUC of 0.996 (95\% $\mathrm{CI}=0.987-1.000$ ) (Figure 4B) in the training set. Moreover, it could effectively separate the blinded samples in the testing set with an AUC of 0.994 (95\% CI $=0.982-1.000)$ (Figure 4C). These results demonstrated that this simplified biomarker panel had an excellent diagnostic performance in discriminating $\mathrm{dHB}$ subjects from $\mathrm{HB}$ subjects.

\section{Discussion}

In this work, we used a dual platform approach (NMR spectroscopy and GC-MS) to further study the divergent urinary metabolic phenotypes between $\mathrm{dHB}$ subjects and HB subjects. Finally, we identified 21 important urinary metabolites responsible for distinguishing $\mathrm{dHB}$ subjects from $\mathrm{HB}$ subjects. Pathway analysis found the significantly affected glyoxylate and dicarboxylate metabolism and glycine, serine, and threonine metabolism. A simplified biomarker panel consisting of $\alpha$-hydroxyisobutyric acid, hippuric acid, azelaic acid, isobutyric acid, malonic acid, levulinic acid, and phenylacetylglycine was identified. This panel had an excellent diagnostic performance in discriminating $\mathrm{dHB}$ subjects from $\mathrm{HB}$ subjects. Moreover, compared to the potential panels identified by single metabolomic platform, ${ }^{12,13}$ this panel had a higher accuracy in diagnosing depression in $\mathrm{HBV}$-infected patients. These results demonstrated that the complementary nature of NMR spectroscopy and GC-MS could provide a better and more specific
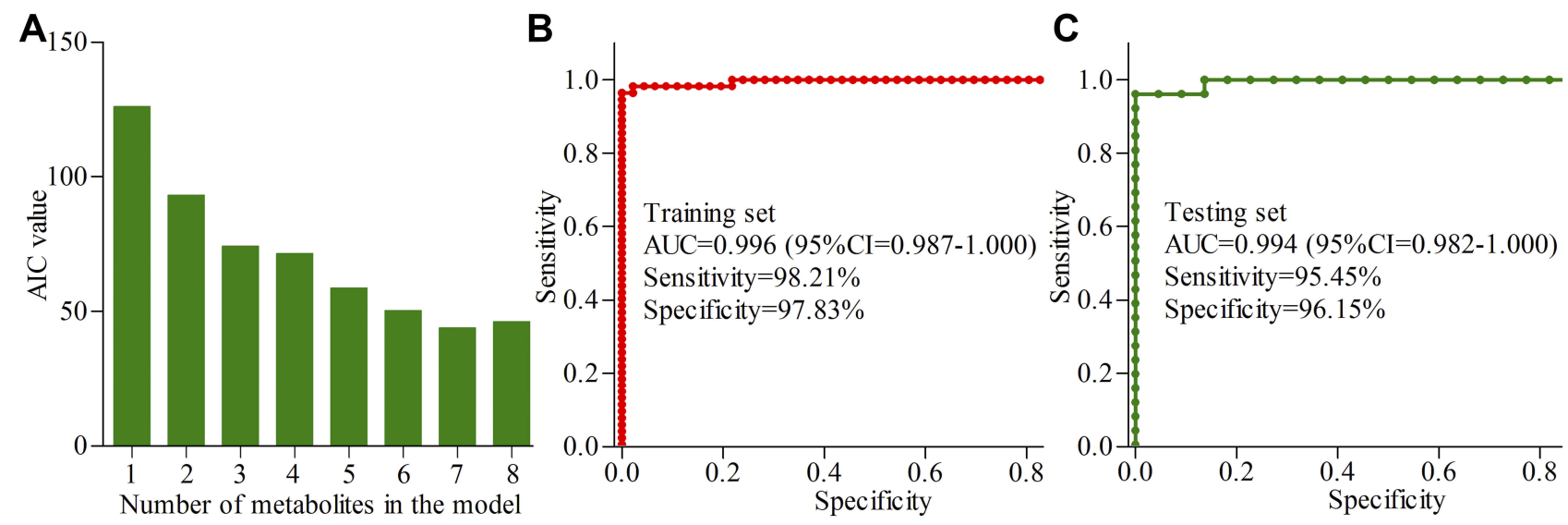

Figure 4 Simplified biomarker panel identification and validation: (A) the bar represents the value of AIC in each model, and the AIC value was minimal when there were seven important urinary metabolites in the logistic-regression model; (B) AUC value in the training set; (C) AUC value in the testing set. 
panel in diagnosing $\mathrm{dHB}$ subjects than any single technique in isolation.

Previous study reported that the multivariate analysis was superior to univariate analysis in finding the potential significance of subtle metabolic differences between different groups. ${ }^{19}$ In our previous metabolic studies, some metabolites were not found to be significantly changed by univariate analysis, but still identified as differential metabolites by multivariate analysis. ${ }^{13,20}$ In this study, the significantly changed levels of 2,4-dihydroxypyrimidine, malonic acid, methylmalonic acid, ethanolamine, phenylacetylglycine, and hydroxylamine were not validated by univariate analysis. However, the built OPLS-DA model still viewed these metabolites as differential metabolites. This was because the addition of these metabolites into the OPLS-DA model could significantly improve its discrimination power.

Depression is a complex disease and its pathogenesis is still not very clear. ${ }^{21-23}$ Our previous study found that the disturbance of gut microbiota might be a contributory factor to the development of depression. ${ }^{24-26}$ Meanwhile, we found that some differential metabolites in depressed subjects, such as usobutyric acid, phenylacetylglycine, p-hydroxyphenylacetic acid, hippuric acid, and dimethylglycine, were the metabolic byproducts of gut microbiota. ${ }^{27}$ Interestingly, these metabolites were also identified as important metabolites in the present study for diagnosing depression in HBVinfected patients. Moreover, previous studies have reported that there was a close relationship between HBV-induced chronic liver disease and gut microbiota. ${ }^{28,29}$ Therefore, future studies were needed to further find out the relationship between gut microbiota and our identified important metabolites, which might be helpful for elucidating the pathogenesis of depression in $\mathrm{HBV}$-infected patients.

Limitations of this study should be mentioned here. Firstly, the number of dHB subjects or HB subjects was relatively small; Large-scale studies were needed to confirm our findings. Secondly, we did not assess the fibrosis stage in the recruited patients; it might be interesting to study whether our findings were appropriate for HBVinfected patients with different stages of liver fibrosis. Thirdly, only urine sample was used here; future studies should collect other biosamples, such as plasma, to further investigate to ensure that the biomarkers identified in this study were relevant to the pathogenesis of depression in HBV-infected patients.

In conclusion, using a dual platform metabonomic approach, we identified 21 important urinary metabolites responsible for distinguishing $\mathrm{dHB}$ subjects from $\mathrm{HB}$ subjects and obtained a simplified biomarker panel consisting of 7 metabolites. This panel could differentiate dHB subjects from HB subjects with a higher accuracy than our previous panels did. These results suggested that the complementary nature of NMR spectroscopy and GCMS could identify a more comprehensive metabolite panel than any single technique in isolation.

\section{Acknowledgments}

This work was supported by the Natural Science Foundation Project of China (81701360, 81601207, 81701477), the Chongqing Science \& Technology Commission (cstc2017jcyjAX0377), the Chongqing Yuzhong District Science \& Technology Commission (20190115), and supported by the fund from the Joint International Research Laboratory of Reproduction \& Development, Institute of Life Sciences, Chongqing Medical University, Chongqing, China, Yongchuan Hospital Foundation of Chongqing Medical University (YJYJ201501, YJJC201911) and also supported by the Scientific Research and Innovation Experiment Project of Chongqing Medical University (CXSY201862, CXSY201863). Liang Fang and Jian-jun Chen are co-senior authors for this study.

\section{Disclosure}

The authors declare no financial or other conflicts of interest in this work.

\section{References}

1. Wu Y, Jin A, Xie G, et al. The 20 most important and most preventable health problems of China: a Delphi Consultation of Chinese Experts. Am J Public Health. 2018;108(12):1592-1598. doi:10.2105/ AJPH.2018.304684

2. Yuen MF, Chen DS, Dusheiko GM, et al. Hepatitis B virus infection. Nat Rev Dis Primers. 2018;4:18035. doi:10.1038/nrdp.2018.35

3. Mirabdolhagh Hazaveh M, Dormohammadi Toosi T, Nasiri Toosi M, Tavakoli A, Shahbazi F. Prevalence and severity of depression in chronic viral hepatitis in Iran. Gastroenterol Rep (Oxf). 2015;3 (3):234-237. doi:10.1093/gastro/gou091

4. Keskin G, Gümüs AB, Orgun F. Quality of life, depression, and anxiety among hepatitis B patients. Gastroenterol Nurs. 2013;36 (5):346-356. doi:10.1097/SGA.0b013e3182a788cc

5. Duan Z, Kong Y, Zhang J, Guo H. Psychological comorbidities in Chinese patients with acute-on-chronic liver failure. Gen Hosp Psychiatry. 2012;34 (3):276-281. doi:10.1016/j.genhosppsych.2011.11.012

6. Huang X, Zhang H, Qu C, Liu Y, Bian C, Xu Y. Depression and insomnia are closely associated with thyroid hormone levels in chronic hepatitis B. Med Sci Monit. 2019;25:2672-2678. doi:10.12659/MSM.914444

7. Chen JW, Xie SQ. Agomelatine versus paroxetine in treating depressive and anxiety symptoms in patients with chronic kidney disease. Neuropsychiatr Dis Treat. 2018;14:547-552. doi:10.2147/NDT.S159636

8. Chen JJ, Xie J, Zeng L, Zhou CJ, Zheng P, Xie P. Urinary metabolite signature in bipolar disorder patients during depressive episode. Aging (Albany NY). 2019;11(3):1008-1018. doi:10.18632/aging.101805

9. Lai CH. Promising neuroimaging biomarkers in depression. Psychiatry Investig. 2019;16(9):662-670. doi:10.30773/pi.2019.07.25.2 
10. Carboni L, McCarthy DJ, Delafont B, et al. Biomarkers for response in major depression: comparing paroxetine and venlafaxine from two randomised placebo-controlled clinical studies. Transl Psychiatry. 2019;9(1):182. doi:10.1038/s41398-019-0521-7

11. Pan JX, Xia JJ, Deng FL, et al. Diagnosis of major depressive disorder based on changes in multiple plasma neurotransmitters: a targeted metabolomics study. Transl Psychiatry. 2018;8(1):130. doi:10.1038/s41398-018-0183-x

12. Hou LJ, Wang HW, Wei XX, et al. Urinary metabonomics for diagnosis of depression in hepatitis B virus-infected patients. Iran Red Crescent Med J. 2015;17(4):e27359. doi:10.5812/ircmj.17(4)2015.27359

13. Hou L, Wei X, Zhuo Y, et al. GC-MS-based metabolomics approach to diagnose depression in hepatitis B virus-infected patients with middle or old age. Aging (Albany NY). 2018;10(9):2252-2265. doi:10.18632/aging.101535

14. Williams R, Lenz EM, Wilson AJ, et al. A multi-analytical platform approach to the metabonomic analysis of plasma from normal and Zucker (fa/fa) obese rats. Mol Biosyst. 2006;2(3-4):174-183. doi:10.1039/b516356k

15. Chen J, Bai S-J, Li W, et al. Urinary biomarker panel for diagnosing patients with depression and anxiety disorders. Transl Psychiatry. 2018;8(1):192. doi:10.1038/s41398-018-0245-0

16. Bouatra S, Aziat F, Mandal R, et al. The human urine metabolome. PLoS One. 2013;8(9):e73076. doi:10.1371/journal.pone.0073076

17. Chen J, Liu Z, Fan S, et al. Combined application of NMR-and GC-MSbased metabonomics yields a superior urinary biomarker panel for bipolar disorder. Sci Rep. 2014;4:5855. doi:10.1038/srep05855

18. Tao R, Huang X, Wang J, Zhang H, Zhang Y, Li M. Proposed diagnostic criteria for internet addiction. Addiction. 2010;105 (3):556-564. doi:10.1111/j.1360-0443.2009.02828.x

19. MacIntyre DA, Jimenez B, Lewintre EJ, et al. Serum metabolome analysis by $1 \mathrm{H}-\mathrm{NMR}$ reveals differences between chronic lymphocytic leukaemia molecular subgroups. Leukemia. 2010;24 (4):788-797. doi:10.1038/leu.2009.295
20. Chen JJ, Xie J, Li WW, et al. Age-specific urinary metabolite signatures and functions in patients with major depressive disorder. Aging (Albany NY). 2019;11(17):6626-6637. doi:10.18632/aging.102133

21. Ménard C, Hodes GE, Russo SJ. Pathogenesis of depression: insights from human and rodent studies. Neuroscience. 2016;321:138-162. doi:10.1016/j.neuroscience.2015.05.053

22. Chen JJ, Xie J, Zeng BH, et al. Absence of gut microbiota affects lipid metabolism in the prefrontal cortex of mice. Neurol Res. 2019;41(12):1104-1112. doi:10.1080/01616412.2019.1675021

23. Wu Y, Zhu B, Chen Z, et al. Prevalence and predisposing factors of depressive symptoms in patients with stable coronary artery disease: a cross-sectional single-center study. Aging (Albany NY). 2019;11 (12):3958-3968. doi:10.18632/aging.102026

24. Chen JJ, He S, Fang L, et al. Age-specific differential changes on gut microbiota composition in patients with major depressive disorder. Aging (Albany NY). 2020;12(3):2764-2776. doi:10.18632/aging.102775

25. Zheng $\mathrm{P}$, Zeng B, Zhou C, et al. Gut microbiome remodeling induces depressive-like behaviors through a pathway mediated by the host's metabolism. Mol Psychiatry. 2016;21(6):786-796. doi:10.1038/ mp. 2016.44

26. Chen JJ, Zheng P, Liu YY, et al. Sex differences in gut microbiota in patients with major depressive disorder. Neuropsychiatr Dis Treat. 2018;14:647-655. doi:10.2147/NDT.S159322

27. Zheng P, Wang Y, Chen L, et al. Identification and validation of urinary metabolite biomarkers for major depressive disorder. Mol Cell Proteomics. 2013;12(1):207-214. doi:10.1074/mcp.M112.021816

28. Kang Y, Cai Y. Gut microbiota and hepatitis-B-virus-induced chronic liver disease: implications for faecal microbiota transplantation therapy. J Hosp Infect. 2017;96(4):342-348. doi:10.1016/j.jhin.2017.04.007

29. Wang J, Wang Y, Zhang X, et al. Gut microbial dysbiosis is associated with altered hepatic functions and serum metabolites in chronic hepatitis B patients. Front Microbiol. 2017;8:2222. doi:10.3389/fmicb.2017. 02222
Diabetes, Metabolic Syndrome and Obesity: Targets and Therapy is an international, peer-reviewed open-access journal committed to the rapid publication of the latest laboratory and clinical findings in the fields of diabetes, metabolic syndrome and obesity research. Original research, review, case reports, hypothesis formation, expert opinion and commentaries are all considered for publication. The manuscript management system is completely online and includes a very quick and fair peer-review system, which is all easy to use. Visit http://www.dovepress.com/testimonials.php to read real quotes from published authors. 